\title{
Les rennes maintiennent la langue nénetse en vie
}

Põhjapõdrad hoiavad neenetsi keele elus

Reindeer Keep the Nenets Language Alive

\section{Laur Vallikivi}

Traducteur : Eva Toulouze

\section{(2) OpenEdition}

12 Journals

Édition électronique

URL : https://journals.openedition.org/efo/2218

DOI : $10.4000 /$ efo. 2218

ISSN : 2275-1947

Éditeur

INALCO

\section{Édition imprimée}

Date de publication : 1 décembre 2013

ISBN : 978-2-343-04446-0

ISSN : 0071-2051

\section{Référence électronique}

Laur Vallikivi, « Les rennes maintiennent la langue nénetse en vie », Études finno-ougriennes [En ligne],

45 | 2013, mis en ligne le 03 février 2015, consulté le 08 juillet 2021. URL : http://

journals.openedition.org/efo/2218; DOI : https://doi.org/10.4000/efo.2218

Ce document a été généré automatiquement le 8 juillet 2021.

\section{(c) (i) (8)}

Études finno-ougriennes est mis à disposition selon les termes de la Licence Creative Commons Attribution - Pas d'Utilisation Commerciale 4.0 International. 


\title{
Les rennes maintiennent la langue nénetse en vie
}

\author{
Põhjapõdrad hoiavad neenetsi keele elus \\ Reindeer Keep the Nenets Language Alive
}

Laur Vallikivi

Traduction : Eva Toulouze

\section{Introduction}

1 Les Nenetses sont souvent présentés comme un "phénomène »; en effet, comparés aux autres " petits $^{1}$ » peuples autochtones de Sibérie, ce sont eux qui semblent avoir le mieux conservé le mode de vie de leurs ancêtres. Différentes causes ont été alléguées, depuis les particularités culturelles (Golovnev, Osherenko 1999) jusqu'aux spécificités de la politique régionale (Stammler 2005). Le tableau n'est pourtant pas aussi homogène qu'on pourrait le penser, puisqu'on rencontre sur l'immense aire couverte par les Nénetses, de la péninsule de Kanin jusqu'à celle de Tajmyr, bien des scénarios différents. Il est vrai que le nombre global des Nénetses est en augmentation constante $^{2}$. Mais qu'en est-il de la langue? Si elle s'est bien maintenue pendant longtemps, sa maîtrise est aujourd'hui, d'après les statistiques, en baisse très rapide : en moins de dix ans, le nénetse a perdu près d'un tiers de ses locuteurs ${ }^{3}$. Comme partout dans le Nord, la tendance générale est au déclin de la langue dans les villages et dans les villes (qui représentent pour les Nénetses un milieu de vie relativement neuf) et à son maintien dans la toundra.

2 Mais les chiffres et les tendances représentent toujours une moyenne, qui peut recouvrir des phénomènes divers, voire contradictoires, et des vécus différents. Le but de cet article est de montrer, par une description ethnographique, que le mode de vie fondé sur l'élevage du renne et sur le nomadisme maintient vivant l'usage du nénetse, alors que la sédentarisation conduit, lentement mais sûrement, vers un monolinguisme russe $^{4}$. Les nomades nénetses sont flexibles dans les choix découlant de leur idéologie 
linguistique : leur rapport avec tel ou tel environnement physique ou social, avec telle ou telle activité physique ou sociale, favorise le mouvement entre différentes langues.

J'ai fait des terrains chez les Nénetses européens entre 1999 et 2012, en tout pendant dix-sept mois, dont une année d'affilée dans les communautés d'éleveurs de rennes non soviétisées (Vallikivi 2003). Dans cet article, je rentrerai un peu dans le détail, sur la base de l'expérience de deux types de communautés qui ont bien conservé la maitrise de la langue, mais qui sont en train de vivre des mutations extrêmement rapides - du monolinguisme au bilinguisme, du bilinguisme au trilinguisme et d'un bilinguisme à un autre.

4 Je vais me concentrer sur deux types de communautés rurales, qui ont maintenu intégralement ou partiellement le mode de vie nomade. Il s'agit tout d'abord de ceux qu'on appelle les "Nénetses kolkhoziens " ${ }^{5}$ : ce sont ceux - ils représentent la majorité absolue - qui ont été « soviétisés » et qui, depuis les années 1920-1950, sont inclus dans les structures officielles (kolkhozes, sovkhozes, école, armée). Les éleveurs de rennes kolkhoziens ont été eux aussi intégrés de diverses manières dans la société soviétique, avec des degrés de soviétisation, là encore, variables. Les plus intégrés étaient ceux qui ne passaient qu'une partie de leur temps dans la toundra sans leurs familles, les moins intégrés ceux qui migraient avec leurs familles, surtout ceux relevant de la catégorie des chasseurs.

5 Le deuxième type concerne une toute petite minorité, ceux qui ont échappé à la soviétisation, c'est-à-dire des Nénetses éleveurs de rennes privés. À la fin de la période soviétique, ils étaient plusieurs centaines. Leur situation représentait une anomalie dans la société soviétique, même dans le Nord. Pour les structures étatiques, ils n'existaient pas, ne figuraient sur aucune liste, n'avaient pas de documents d'identité et n'étaient donc concernés ni par l'école ni par l'armée ( $\mathrm{cf}$. pour plus de détails Vallikivi 2003, 2009). Leur existence était possible avant tout en raison de leur volonté de rester indépendants - à l'époque stalinienne, la plupart des Nénetses partageaient sans doute ce souhait, sans pour autant parvenir à échapper au réseau soviétique.

6 Je vais parler ici de deux groupes d'éleveurs de rennes privés non soviétisés: la frontière entre eux n'est pas imperméable, mais ils se distinguent par des conditions de vie différentes. Les Nénetses de Jamb-to (environ deux cents personnes) migrent dans la toundra dans le district autonome nénetse et passent l'été au bord de la mer; le groupe des Nénetses de l'Oural (plus de trois cents) passe l'été dans les montagnes ou à proximité de celles-ci, et migre entre la République komie et le district autonome iamalo-nénetse. Depuis les années 1990, ces deux groupes ne sont plus complètement isolés des structures étatiques: ils ont progressivement acquis une existence dans la Fédération de Russie, dans la mesure où désormais la loi permet à nouveau la propriété privée.

7 Naturellement, la situation linguistique diffère considérablement entre les Nénetses soviétisés et les Nénetses non soviétisés (mais également au sein de ces deux catégories). Les vingt ou trente dernières années ont vu une certaine réduction de cet écart, tout en introduisant, avec de nouveaux phénomènes comme la conversion au christianisme, des différences parfois surprenantes. 


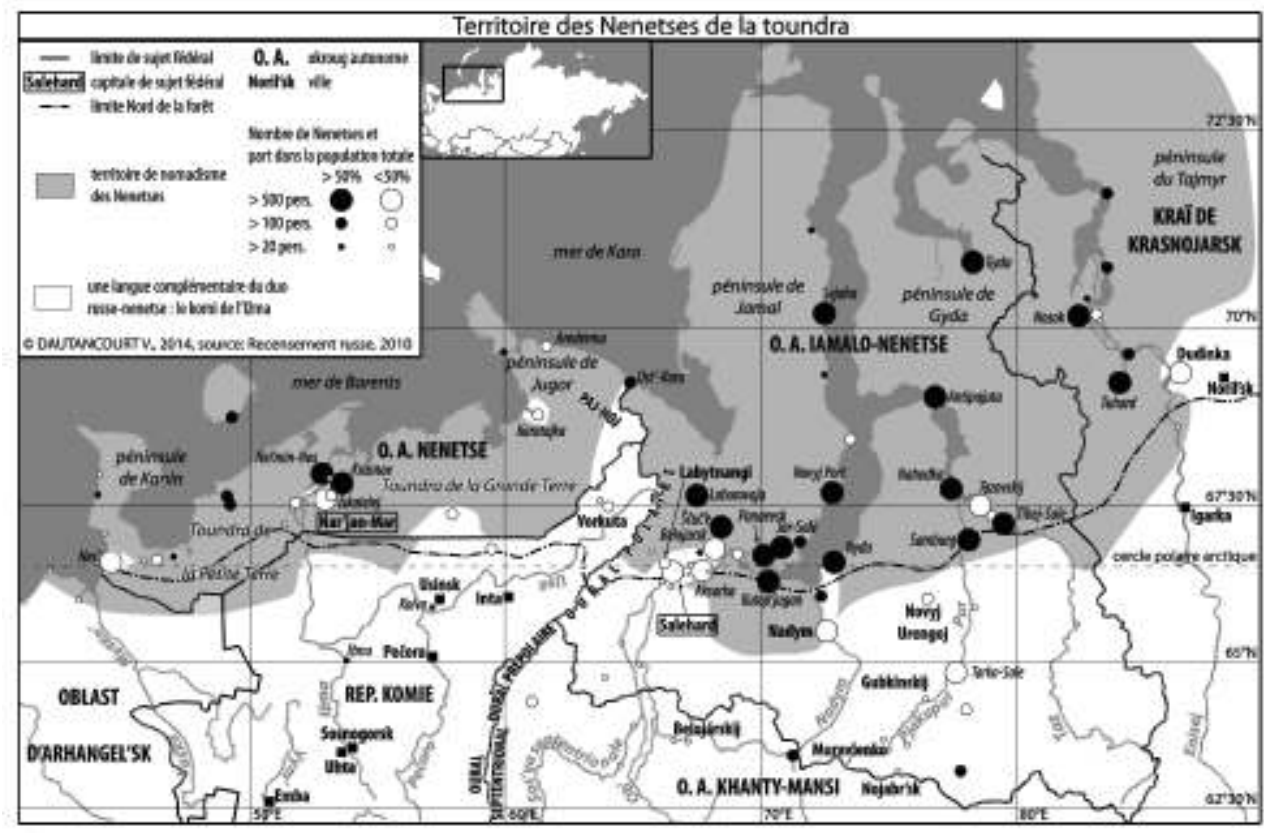

Source : Recensement russe 2010

Copyright: V. Dautancourt, 2014

\section{Les Nénetses kolkhoziens}

La première fois que je suis allé chez les éleveurs de rennes nénetses kolkhoziens, en 1999, dans la toundra de la Petite Terre (Njudja Ia) dans le district autonome nénetse, j'ai rencontré des Nénetses d'âge moyen qui étaient entièrement bilingues russe-nénetse. Entre eux, ces hommes âgés de quarante à cinquante ans parlaient nénetse, alors que le jeune éleveur de vingt-trois ans qui était avec eux n'en était pas capable, même s'il comprenait ce que les autres disaient. Il était rare de voir dans la toundra des femmes, des enfants et des personnes âgées ; l'épouse de l'éleveur le plus âgé était la seule femme du groupe et remplissait la fonction de "travailleuse de tente ${ }^{6} »$. En effet, depuis les années 1960, parmi les Nénetses les plus occidentaux, l'élevage des rennes a été réformé de manière à ce que seuls les éleveurs, donc les hommes, vivent dans la toundra ; les femmes, les enfants et les vieillards ont été relogés au village. Les hommes travaillent dans la toundra avec les rennes suivant un système d'alternance: ils passent un certain temps (en général un ou deux mois) dans la toundra, et autant de temps au village. Dans le district autonome nénetse, les autorités locales étaient particulièrement zélées dans leur observation des instructions de Moscou : c'est ainsi que dans les années 1980 les migrations de familles entières dans le cadre du système kolkhozien restèrent marginales ${ }^{7}$.

9 Quand j'ai passé quelque temps avec une brigade d'éleveurs de rennes, il y avait également une femme un peu plus jeune, institutrice à l'école, qui passait ses vacances d'été dans la toundra avec son mari, éleveur de rennes de son métier, et ses deux fils de huit et de dix ans ; les enfants ne parlent plus nénetse, contrairement à leurs parents : ce sont des Nénetses russophones monolingues. Majoritairement, la jeune génération, celle qui a autour de vingt ans, ne parlait pas non plus nénetse, même si certains le 
comprenaient ; parmi ceux qui ont dix ans de moins, il n'en reste plus que quelquesuns $^{8}$.

En effet, au village, le nénetse recule, notamment parmi les jeunes : à l'école, seul le russe est utilisé en tant que langue d'enseignement, alors que le nénetse est enseigné deux ou trois heures par semaine. Les habitants vivent dans l'espace médiatique russe et le prestige du nénetse - comme en général celui des langues autochtones à l'époque soviétique - est extrêmement bas9.

11 Dans ce cas précis, le prestige de la langue n'a guère bénéficié de ce que les villages sont principalement habités par des Nénetses. Ces éleveurs étaient officiellement inscrits au village de Nel'min-Nos, où ils ont des maisons. Nel'min-Nos est un village dit «national ${ }^{10}$ ». En 2002, d'après le recensement, il y avait à Nel'min-Nos 969 habitants, dont 885 Nénetses. Parmi ces derniers, 883 avaient noté qu'ils maitrisaient le russe, et 424 avaient affirmé maîtriser le nénetse en tant que première « langue autre " ${ }^{11}$.

12 Telle est la situation dans le village de Nel'min-Nos. N'oublions pas que le district autonome nénetse est le plus occidental et que sa population a été en contact avec les Russes (et avec les Komis) bien avant les autres. Mais c'est surtout l'activité réformatrice intense des autorités, à l'époque soviétique, qui a conduit au recul de la langue dans ce district. En même temps, dans les régions plus orientales, il existe des espaces où les Nénetses « kolkhoziens " maitrisent la langue de manière plus active et où même les jeunes générations sont presque intégralement nénetsophones, par exemple les péninsules de Jamal, Gyda, Tajmyr (cf. Krivonogov 1997 ; Volžanina 2010). Et ce tout d'abord en raison de la migration avec les rennes par familles entières.

\section{Les éleveurs de rennes non soviétisés}

13 Au cours de mon deuxième terrain, en 2000 , je suis allé quatre cent kilomètres plus à l'est, chez des éleveurs de rennes non soviétisés, dans la communauté de Jamb-to, où les hommes d'âge moyen, mais aussi les plus jeunes, les femmes et les enfants, qui vivent en famille dans la toundra, utilisent le nénetse quotidiennement : c'est leur toute première langue. Encore dernièrement, au milieu des années 1990, presque tous les membres de ces communautés n'avaient du russe qu'une connaissance minime ou ne le savaient pas du tout ${ }^{12}$. Les connaissances des femmes sont toujours très faibles, les hommes maîtrisent aujourd'hui un peu mieux le russe, surtout la langue des relations commerciales, du marchandage.

14 Il existe aujourd'hui deux communautés non soviétisées (Jamb-to et Oural). Leurs contacts avec le milieu russophone ont été limités, et ce de manière délibérée, car pendant des décennies elles ont évité les Russes ${ }^{13}$, qu'elles appellent lutsa. Il y a à cela des raisons historiques : la politique soviétique a conduit les Nénetses à craindre les autorités. Dans le cadre de la collectivisation, ces dernières confisquèrent les rennes et formèrent des kolkhozes. Cette politique se heurta au mécontentement de la population, qui tenta de diverses manières d'échapper à toutes les nouvelles contraintes - y compris la scolarisation des enfants et le service militaire.

15 En 1943, une grande partie des Nénetses de la région formèrent ce qu'en nénetse on appelle une mandalada, c'est-à-dire qu'ils se rassemblèrent pour discuter de leur avenir et allèrent jusqu'à reprendre leurs rennes. Ce mouvement déboucha sur une lutte armée et fut durement réprimé (Vallikivi 2005). Beaucoup des éleveurs qui ne se 
laissèrent pas soviétiser comptent parmi les descendants directs ou indirects de ceux qui se sont opposés aux autorités dans les années 1940. Si ces groupes de Nénetses ont voulu éviter tout contact, après la période stalinienne les autorités n'ont pas investi beaucoup d'énergie dans la recherche des derniers éleveurs indépendants, d'autant que ceux-ci étaient assez pauvres et possédaient peu de rennes. Les contacts avec le monde extérieur à la toundra étaient limités, mais non inexistants, puisque le commerce continuait dans une certaine mesure.

Cette région était fréquentée aussi par des éleveurs de rennes komis; une partie des Nenetses avait été «komisée » et était devenue komiphone, il y avait des mariages entre les deux communautés, ce qui fait que la langue komie était présente, notamment dans le groupe de Jamb-to (cf. ci-dessous). Autrement, la langue principale de communication était le nénetse pour toutes les générations.

Un tournant fut amorcé dans les années 1990 dans le domaine linguistique; il se poursuit encore de nos jours. Plusieurs phénomènes concomitants confluent : l'État témoigne d'un intérêt nouveau pour les groupes marginalisés; certains membres du groupe manifestent un intérêt particulier pour le monde russe et font preuve d'une volonté de démarginalisation qui rejoint les initiatives de l'État; cette démarginalisation par rapport au monde russe passera en partie par la conversion de quelques membres du groupe au christianisme évangélique sous la forme de leur rattachement aux baptistes non enregistrés ${ }^{14}$, qui convertiront dans les années suivantes environ la moitié des deux groupes. Tout ceci a une incidence considérable sur les pratiques linguistiques.

\section{La démarginalisation par rapport au monde russe : l'école}

Pendant les années soviétiques, les Nénetses de ces deux groupes n'allaient pas à l'école. Mais il y eut des exceptions. Par exemple deux enfants de Jamb-to, dans les années 1970, se retrouvèrent à l'école un ou deux ans ${ }^{15}$. Cette expérience, assez dure, leur apporta une connaissance de la langue russe bien meilleure que celle des autres et les encouragea à devenir des intermédiaires entre leur groupe et le monde russe; ce seront aussi les premiers convertis baptistes. Mais c'est surtout à partir des années 1990 que l'école joue massivement un rôle dans la vie du groupe, aussi bien pour les enfants que pour les adultes.

\section{L'école-internat}

L'école-internat est l'outil principal, sinon unique, de scolarisation des enfants dans les régions du nord de la Russie. Elle a été développée à l'époque soviétique et présentée comme un grand résultat de la politique de soviétisation. Les enfants sont rassemblés dans un établissement où ils passent l'année scolaire isolés de leurs familles, qui se déplacent dans la toundra ou dans la taïga dans lesquelles ils retournent en général pendant les vacances. Si, au début de la période soviétique, dans les années 1920-1930, les autorités encourageaient l'utilisation de la langue vernaculaire dans les écoles, depuis la fin des années 1950 la politique officielle a changé et l'enseignement des langues vernaculaires a commencé à être vu comme nuisible à l'apprentissage du russe. De ce fait, même les conversations des enfants entre eux dans leur langue furent interdites dans les écoles (Liarskaya 2013, p. 163-4; cf. aussi Bloch 2004; Toulouze 1999). Or les enfants des éleveurs privés échappèrent à cette politique de 
russification linguistique intense, dans la mesure où leurs contacts avec le système d'éducation datent pour la plupart de l'époque postsoviétique, où la russification prend des formes moins agressives.

Au milieu des années 1990, dans le groupe de Jamb-to, les autorités ont commencé à faire pression pour que les enfants aillent à l'école : il a fallu du temps pour que la majorité des parents accepte, car ils avaient vécu dans la peur qu'on leur prenne de force leurs enfants, comme c'était de rigueur à l'époque soviétique. Mais aujourd'hui, la quasi-totalité des enfants est scolarisée, au moins pendant quelques années (en général trois ou quatre ans). Dans le groupe de l'Oural, ce sont certains parents, surtout ceux dont les troupeaux sont petits, qui, un peu plus tard, à la fin des années 1990, prennent l'initiative de mettre leurs enfants pour quelques années à l'école du village de Sovetskij à proximité de Vorkuta, en partie pour leur assurer nourriture et habillement, aussi longtemps qu'ils ne sont pas, de toute manière, en mesure d'aider les parents.

21 À l'école, l'enseignement est très largement russophone, alors que les enfants nénetses arrivent sans parler russe. À l'école de Karatajka, où vont les enfants de Jamb-to, ils suivent pendant un an des classes dites d'adaptation pour apprendre le russe avec un enseignant parlant nénetse ${ }^{16}$; par la suite, le nénetse reste dans les curricula en tant que matière et est enseigné comme une langue étrangère quelques heures par semaine (L. Taleeva 2002 : p. 10). En revanche dans l'école de Sovetskij, aucune enseignante ou puéricultrice ne parle nénetse (il y en avait une, mais elle n'est restée que peu de temps, cf. Drama 2000). Ce qui veut dire qu'au début, il n'y a aucune langue de communication entre les élèves et leurs institutrices, ce qui est une expérience difficile pour les deux.

\section{Le projet d'école d'été}

Entre 1997 et 2007, un projet d'école nomade a été lancé pour les Nenetses de Jamb-to par l'anthropologue norvégien Ivar Bjørklund ${ }^{17}$ et financé par le projet Barents, avec des fonds norvégiens et russes. C'est ce qu'on a appelé l'école nomade d'été ${ }^{18}$. Pendant un mois ou un mois et demi, plusieurs enseignants (quatre la plupart du temps), des Nenetses de Nar'jan-Mar, vivaient dans les tentes avec les éleveurs de rennes et enseignaient le russe, les mathématiques et "le monde environnant", matière qui, conformément au programme des écoles primaires russes, traite de l'homme, de la nature, de la société et de la technologie. Ces enseignants, bien qu'étant Nénetses, étaient originaires de la ville, et la plupart n'avaient pas l'expérience de la vie dans la toundra; ces cours leur ont donné l'occasion de se voir comme introduisant le monde extérieur auprès des éleveurs indépendants de Jamb-to (Bjørklund 2000; Žuravleva 2000).

23 Ce projet avait comme objectif d'alphabétiser les adultes, qui étaient jusqu'alors illettrés, émulant la campagne de liquidation de l'analphabétisme (likbez) du début de la période soviétique; il entendait aussi proposer une alternative à l'internat, instruisant les enfants dans leur propre milieu. Les résultats se sont avérés mitigés : les adultes motivés, ceux qui voulaient apprendre à lire la Bible, autrement dit la plupart des baptistes, ont été effectivement alphabétisés; les autres, aux dires des enseignants, trouvaient toujours d'autres priorités. Pour beaucoup, une scolarisation excessive représentait un danger : ils avaient peur que les enfants s'éloignent de la vie dans la toundra. Comme l'a dit un père à une institutrice de l'école d'été : « Qui va conduire la 
caravane, monter la tente, confectionner les vêtements, nous aider? Si ma fille fait de longues études et vit longtemps à l'internat, elle deviendra comme toi. Car toi, tu as perdu l'habitude de la vie dans la toundra, tu ne sais pas conduire un attelage, tu as peur des rennes et même l'été, tu es frigorifiée. Et sans doute, tu ne sais pas coudre des vêtements d'hiver. Tu es devenue une habenye (une Nénetse russifiée). Dans la toundra, on n'a pas besoin de femmes comme toi » (M. Taleeva 2010).

Le projet s'est arrêté en 2007 : les autorités ont estimé qu'il avait réalisé ses objectifs et sans doute les financements ont-ils été interrompus.

\section{L'école « de l'Église »}

Au milieu des années 1990, un missionnaire russophone ukrainien venu de Vorkuta, Nikolaj, a commencé à rendre visite aux éleveurs privés de Jamb-to - invité par un jeune Nénetse qui avait eu l'expérience de l'école dans les années 1970 (cf. plus haut). Son message était que la voie vers le salut est la foi et la communication avec Dieu. Bien que toutes les langues soient aptes à cela, le russe est une langue sûre, éprouvée, car il existe une traduction russe des Écritures "contrôlée par le Saint-Esprit». Les missionnaires, qui n'ont pas appris le nénetse, communiquent avec les Nénetses qui ne parlent pas russe par l'intermédiaire d'un interprète; mais ils les encouragent à apprendre le russe ainsi qu'à le lire, ce qui est indispensable pour pouvoir s'inscrire dans la plus large communauté de tous les croyants (bien sûr, il est question des baptistes appartenant à leur Union).

En 2001, pour contrebalancer l'effet nocif de l'école officielle et l'hostilité des enseignants nénetses de l'école d'été, Nikolaj a organisé à Vorkuta un internat privé pour les enfants nénetses, situé à proximité de la maison de prières de la ville. Les coûts de fonctionnement étaient couverts par les familles nénetses, qui vendaient de la viande au marché. La première année, l'école accueillit douze enfants. La deuxième année leur nombre monta jusqu'à vingt. Au bout de deux ans, le projet fut abandonné, car les difficultés matérielles et les questions d'organisation devinrent trop pesantes. Par ailleurs, l'école n'était pas officiellement reconnue. L'accumulation de problèmes a conduit l'Église à la fermer.

Néanmoins, des jeunes femmes baptistes venues d'Ukraine, de Saint-Pétersbourg et d'ailleurs se rendirent auprès des croyants dans la toundra pour leur apprendre à lire, à chanter et à connaître les évangiles. Comme dans le cas des institutrices nénetses, les enseignants de l'Église passaient d'une famille à l'autre et restaient avec chacune deux ou trois semaines. Mais à la différence du programme de l'école d'été, l'enseignement était en russe et reposait sur la Bible.

La langue russe a acquis progressivement de plus en plus de prestige auprès des Nénetses: la maîtrise du russe a toujours été appréciée, ne serait-ce que pour des raisons pratiques; mais maintenant, l'intégration dans la société russe a augmenté son caractère indispensable ; par ailleurs, la pénétration du christianisme évangélique est sans doute aujourd'hui le facteur principal de russification, car le russe est la langue de l'Église et de la Bible. En même temps, il ne faut pas négliger la nécessité de communiquer aussi bien avec les marchands qu'avec les administrations, qui gèrent les retraites et les allocations familiales.

À l'école, le russe pénètre même dans les conversations entre enfants nénetses (M. Taleeva 2010). Les enfants, qui aident parfois leurs parents à régler des affaires en 
russe, ont l'impression que grâce à cette langue, ils comptent dans leur famille. Après leur retour dans la toundra, la plupart retournent au nénetse, mais certains individus gardent le russe parmi leurs langues de communication, notamment dans les familles de convertis. Au cours de mes travaux de terrain, je n'ai jamais entendu personne faire des reproches aux enfants qui de temps en temps parlent russe entre eux. En même temps, les rêves des enfants sont souvent liés au monde russe, ce qui inquiète les parents, qui ne souhaitent pas que leurs enfants abandonnent la vie avec les rennes (Vallikivi, à paraître).

\section{La démarginalisation par rapport au monde russe : le christianisme}

La moitié environ des Nénetses non soviétisés a été convertie au baptisme (dans l'Oural, on trouve aussi quelques familles pentecôtistes). Ceux qui sont restés adeptes de la vision traditionnelle du monde nénetse sont moins exposés que les autres à la langue russe et, dans leur vie, le nénetse reste quasiment exclusif. Certes, le russe demeure la langue du commerce et la langue de l'école pour les plus jeunes. Mais le prestige et la nécessité du russe sont moins présents au quotidien. Je vais me concentrer ici sur ceux qui ont été convertis, et qui sont largement exposés par ce canal à la présence du russe, même si celle-ci n'a guère abouti à l'élimination du nénetse.

31 Le russe est avant tout présent par le truchement des missionnaires. Ceux-ci sont russophones ${ }^{19}$. Pour eux, le russe, la connaissance de cette langue est le moyen principal qui permet aux croyants de se développer dans la foi, de devenir de bons croyants et d'entrer dans l'Église universelle, ce qui passe par la lecture, la prédication et la conversation. Les baptistes non enregistrés sont exclusivistes: eux seuls sont porteurs de salut et la seule Église universelle légitime est la leur, et, même si elle est pluriethnique et qu'on utilise dans certains cas d'autres langues, le russe est ressenti comme le dénominateur commun indispensable. Le principal missionnaire de Vorkuta a même affirmé que l'Union soviétique, malgré tous ses vices, faisait partie du plan divin d'unification des nations, et le fait que le régime ait amené toutes ses ethnies à apprendre le russe est un pas en avant dans la perspective de leur salut. C'est pourquoi apprendre le nénetse et traduire dans cette langue n'est pas pour eux une priorité, même s'ils n'hésitent pas à diffuser des textes religieux traduits par d'autres. De plus, les missionnaires considèrent que les langues locales ne sont pas suffisamment développées ${ }^{20}$. Ils rejettent également la pratique du chant et de la narration en nénetse, ce qui touche donc les histoires les plus anciennes. Ainsi, dans les tentes des chrétiens, les genres oraux traditionnels ont cessé d'être pratiqués.

Ils sont remplacés par une parole nouvelle, qui joue un rôle central dans les pratiques religieuses baptistes. La foi est affirmée, confirmée et renforcée par le comportement, mais dans le comportement, le principal est la proclamation de la foi à voix haute, le témoignage et la prière publics. Ainsi les Nénetses sont-ils amenés - ce qui constitue une mutation culturelle considérable dans leur pratique linguistique et dans leur conception de la langue - à s'exprimer oralement d'une manière neuve, inhabituelle. Ils sont tenus de prier devant la communauté. $\mathrm{Si}$, dans la toundra, ils le font en nénetse, quand les missionnaires sont là, ceux qui en sont capables prient en russe. Les autres prient en nénetse, mais leurs prières sont traduites en russe par les deux ou trois croyants qui sont en mesure de le faire. Bien que l'activité rituelle soit en russe, un 
certain nombre d'activités tournées vers les Nénetses, par exemple des services religieux en ville, sont entièrement traduites.

En fait, la maîtrise du russe est très largement question de génération. La jeune génération, adolescents et enfants, joue un rôle central dans l'intégration de leurs aînés : souvent, ce sont ces jeunes qui aident leurs parents à comprendre la Bible, ils traduisent et expliquent. Parmi les plus âgés, la connaissance du russe est variable: tous les hommes sont, à des degrés divers, capables de marchander au moins à un niveau élémentaire, mais d'autres aujourd'hui ont appris à utiliser le langage religieux (prières, Bible).

De manière générale, le russe a donc pénétré dans un milieu qui lui était plutôt fermé au départ, et le bilinguisme russe-nenets existe aujourd'hui réellement. Les enfants scolarisés sont réellement bilingues, les adultes le sont de manière variable et cela se traduit dans une communication plus large avec le monde extérieur. Mais que se passet-il dans le milieu traditionnel nénetse, dans la toundra, dans les campements d'éleveurs de rennes, quand les missionnaires ne sont pas présents, compte tenu de ces mutations dans le contexte?

\section{Usages des deux langues dans la toundra}

Comme nous l'avons dit, les jeunes qui ont été scolarisés, dont la connaissance du russe est courante, ont parfois tendance à l'utiliser dans la toundra, qui est le milieu nénetse par excellence, en parlant entre eux.

Ils l'utilisent dans des situations ludiques, souvent au deuxième degré, avec une certaine théâtralité. Peut-être ma présence appelle-t-elle dans une certaine mesure l'usage du russe, mais ce n'est pas le seul facteur, car j'ai pu constater le même phénomène dans des cas où je n'étais pas présent au début de l'acte de communication $^{21}$. Il est difficile de proposer des exemples précis, car ce n'est pas le contenu de la communication qui est en cause, mais des indicateurs corollaires perceptibles: le ton, l'attitude, la gestuelle, l'expression du visage, qui fonctionnent comme avec valeur de citation. On a l'impression que ces jeunes Nénetses jouent les Russes, et imitent des formes de communication propres aux Russes, des attitudes et des intonations. Ce n'est pas forcément un phénomène entièrement nouveau. Chez des Nénetses plus âgés, qui n'ont du russe qu'une connaissance minime, il arrive que l'état d'ivresse déclenche une imitation des Russes, une sorte de regard extérieur, distancé et légèrement ridiculisant (cf. Vallikivi, à paraître).

Mais il y a d'autres situations, tout autres que ludiques, qui appellent l'usage du russe de préférence au nénetse. Ce sont les situations qui mettent les Nénetses au contact de « choses » du monde russe. Un exemple parlant, qui s'est répété maintes fois dans mon expérience, est le suivant : quand, dans la toundra, une motoneige doit être réparée et que les jeunes se rassemblent autour pour discuter quoi faire, ils parlent souvent en russe. Il est vrai que la terminologie existe uniquement dans cette langue. Mais dès qu'on se tourne vers les activités en rapport avec les rennes, toute référence au russe disparaît, et les mêmes utilisent uniquement un nénetse non mêlé de russe ${ }^{22}$. De manière plus globale, ceci confirme et précise la tendance générale évoquée en introduction : le nénetse se maintient dans la toundra, même s'il est menacé ailleurs. Or 
dans la toundra, l'élément autour duquel tourne la vie des Nénetses est le renne (Niglas 1997).

Si l'exemple précédent nous montre un comportement spontané et un changement de langue inconscient, dans un contexte de multilinguisme, l'usage de telle ou telle langue peut aussi relever d'un choix stratégique en situation. Par exemple, les éleveurs de rennes komis disent souvent que les Nénetses font semblant de ne pas parler komi pour se mettre en position avantageuse.

\section{Bilinguisme ou trilinguisme?}

Cet exemple nous amène à développer la question de la présence du komi. Pour la commodité de l'exposé, je me suis concentré jusqu'ici sur le bilinguisme russe-nénetse. Il est temps de compliquer le tableau en faisant intervenir une langue que je n'ai encore mentionnée qu'en passant, mais qui joue un rôle important dans cette région.

Comme dans d'autres zones frontalières des territoires nénetses (cf. Laptander dans ce volume), d'autres langues peuvent avoir une certaine présence : dans cette région, c'est le komi ${ }^{23}$. En effet, c'est ici que les Nénetses coexistent avec les Komis les plus septentrionaux, les Komis dits de l'Ižma, autrement dit Iz'vatas. Ce sont les descendants de Komis venus de contrées plus méridionales et entrés en contact avec les Nénetses dans la région où la taïga devient toundra. Ils ont adopté les pratiques et le mode de vie nénetses, notamment l'élevage du renne (cf. Šabaev 2011). Avec le développement d'un élevage de rennes à grande échelle, au XIX ${ }^{e}$ siècle, les Iz'vatas ont rassemblé d'immenses troupeaux, pesant ainsi fortement sur l'économie nénetse de la région et asservissant une partie des Nénetses appauvris. Le poids des Komis a été tel qu'une partie de la population nénetse a été "zyriénisée» et a adopté le komi comme langue de communication. On les appelle les Jaran ${ }^{24}$. Le processus commença dans les années 1830 , quand une partie des Nénetses des forêts européens, ayant perdu leurs rennes, se sédentarisèrent au village de Kolva, dans la toundra de la Grande Terre (Ngarka Ja). Les hommes nénetses prirent pour épouses des Komies des villages environnants (Homič 1976, p. 148 ; Mamadyšskij 1910, p. 65-66) et passèrent entièrement au dialecte komi de l'Ižma (Dolgih 1970, p. 48 ; cf. Istomin 1999). Ces Nénetses sédentarisés de Kolva passèrent ainsi à l'élevage des bovins et à l'horticulture. Cependant, certains retournent à l'élevage du renne à la fin du XIX ${ }^{\mathrm{e}}$ siècle $^{25}$.

41 Babuškin, qui a travaillé pour le recensement polaire soviétique (1926-1927), souligne que dans les années 1930, ceux des Nénetses qui nomadisent dans la zone forestière sont passés dans la communication quotidienne au komi ; ceux qui passent l'hiver dans la toundra parlent le komi, tout en ayant "préservé la pureté de leur langue ${ }^{26}$ » (1930, p. 65). C'est dans les années 1950 que le komi a commencé à dominer vraiment, comme me l'a dit un Nénetse aujourd'hui komiphone, qui travaillait comme chasseur au kolkhoze de Ust'-Kara et qui est maintenant éleveur privé. En revanche, dans la communauté de l'Oural, la connaissance du komi était moins généralisée.

Aujourd'hui, les mariages entre Iz'vatas et Nénetses sont rares ou inexistants. En revanche, il existe des mariages entre Jaran et Nénetses, notamment chez les Nénetses de Jamb-to, ce qui a renforcé la langue komie dans le groupe. En effet, elle y était déjà présente, car les contacts existaient, dans la mesure où les pâturages coïncidaient ou 
étaient limitrophes ${ }^{27}$; comme dans le cas du russe, la connaissance d'un komi au moins élémentaire était répandue à des niveaux divers, sans pour autant être généralisée.

La présence du komi nous oblige à réfléchir sur la dynamique compliquée et variable de la pratique linguistique, même dans la vie d'un seul individu. Quelques exemples de biographies linguistiques permettront de comprendre comment peut fonctionner cette fluidité linguistique.

Je vais me concentrer sur l'expérience d'un frère et d'une sœur, Lena et Kolja, entre lesquels il y a une certaine différence d'âge, quinze ans environ, et qui tous les deux ont épousé des Nénetses de Jamb-to. Petits, les deux enfants parlaient nénetse avec leur mère. Puis le komi, la langue du père, a pris la première place dans la famille. Quand les enfants sont allés à l'école-internat de Ust'-Kara, le kolkhoze Octobre rouge où leurs parents travaillaient en qualité de chasseurs de bêtes à fourrure, est passé au komi ; les enfants se sont retrouvés dans un milieu à dominante komie, mais où le russe était présent. Ils ont perdu le nénetse. Lena a même fait des études de couture dans la région de Moscou, donc en russe. Plus tard, elle a été mariée à Jakov, un Nénetse nénetsophone éleveur de rennes de Jamb-to - elle venant d'un kolkhoze et lui de l'élevage privé. Au début, les mariés n'avaient pas de langue commune. Jakov évoque leurs difficultés de départ, non seulement en raison de la langue, mais aussi parce que Lena n'était pas capable d'effectuer nombre de tâches féminines dans la toundra. Peu à peu, ils ont chacun appris plus ou moins bien la langue de l'autre, c'est-à-dire que Lena a retrouvé le nénetse. Mais souvent chacun parle sa langue. Lena, à l'inverse de son mari, qui parle volontiers komi avec des Komis, est réservée en nénetse, et évite de parler nénetse en compagnie d'autres Nénetses. Jakov a cependant instauré des règles de communication internes à la famille; il considère que les enfants étant "nénetses ", ils doivent parler le nénetse, et même si leur mère leur parle en komi, ils répondent en nénetse.

45 Kolja, le frère cadet de Lena, a aussi commencé avec le nénetse. C'est pendant qu'il était à l'école que le komi a pris la position dominante, de sorte que de retour auprès des parents pour les vacances, il était amené à parler komi avec ses parents et il a oublié le nénetse. Il a épousé dans les années 1990 une femme nénetse vivant dans la toundra, la sœur de Jakov. Si Lena évite de parler nénetse, son frère a très bien appris cette langue en quelques années et c'est sa première langue de communication quotidienne. Il affirme cependant qu'il lui est plus facile de parler komi. De plus, aussi bien Lena que Kolja parlent bien le russe, ce qui fait qu'ils sont tous deux trilingues.

Nous pouvons déduire de ces exemples que le passage d'une langue à l'autre et même des allers et retours entre langues dans la vie d'un individu ne sont pas une exception; je pourrais multiplier les exemples. Dans cette région, les gens sont habitués à ce phénomène : dans une vie, on peut changer de langue, ne serait-ce que pour des raisons pragmatiques. Cela ne veut pourtant pas dire que le rapport aux langues et aux changements de langue soit indifférent. Des jugements de valeur, eux-mêmes divers, parfois individuels, peuvent intervenir. Je prendrai l'exemple d'une famille nénetse qui, pendant quelque temps, vécut dans la même tente qu'une famille komiphone. La petite fille (aujourd'hui une femme de 60 ans, qui m'a raconté cette histoire) utilisait le komi. Un Nénetse lui a demandé un jour pourquoi elle parlait "cette mauvaise langue " (vyvku vada en nenets). D'ailleurs depuis elle n'a parlé que nénetse, dans la mesure où cette situation de coexistence n'a pas été répétée. Mais dans cette coexistence des langues, il y a toujours des plus forts et des plus faibles. Malgré l'exemple ponctuel 
précédent, la tendance actuelle est que, par rapport au komi, le nénetse est ici la langue la moins prestigieuse. La narratrice de ce dernier exemple précise d'ailleurs que dans la tente, quand elle était enfant, les komiphones parlant komi d'un côté de la tente, ses parents, de l'autre côté, s'étaient mis eux aussi à parler komi.

\section{Conclusion}

Derrière les pratiques et les attitudes décrites ici, il nous faut réfléchir à l'idéologie linguistique qui les sous-tend. Une grande partie des communautés finno-ougriennes les plus occidentales et méridionales ont tendance à considérer la langue comme le facteur central dans l'identité (cf. Grünthal, Kovács 2011). Pour les peuples du Nord, le mode de vie joue un rôle tout à fait analogue (cf. Toulouze 2012). On aura compris que ces Nénetses ne sont pas de grands militants de leur langue; ce sont des utilisateurs pragmatiques, pour qui la langue n'a pas forcément le rôle central. Les techniques non verbales sont très présentes: silence, observation, imitation sont au cœur de la transmission, y compris dans l'éducation non formelle des enfants.

Quand on parle de mode de vie, c'est une expression très générale qui peut s'articuler en divers facteurs identifiables. Si l'on est capable de vivre la vie de la toundra, qui est exigeante, la langue n'est pas d'une importance première. Il n'existe pas d'idéologie linguistique qui rattache la langue à l'identité de groupe de manière indélébile. On accorde de l'importance avant tout à d'autres qualités, comme le savoir-faire et les connaissances $^{28}$. L'essentiel semble être le lien maintenu avec les rennes, qui font preuve, dans la préservation de la langue, d'une agentivité certaine.

Cela ne veut pas dire que la langue n'ait aucune fonction identitaire. Cela veut dire que celle-ci est moins absolue qu'ailleurs et qu'elle peut être actualisée ou non suivant la situation. D'ailleurs, de manière générale, bien des auteurs ont souligné le caractère situatif de l'identité ethnique dans cette région du monde (Anderson 2000; Balzer 1999) : suivant le destinataire, pour les Nénetses privés, la manière de s'habiller et la manière de porter la ceinture peuvent aussi jouer un rôle de marqueurs ethniques.

Ainsi, la langue ne marque pas de manière essentielle l'identité des Nénetses russifiés, des lutsa. On appelle lutsa dans l'ensemble de la Sibérie, les porteurs de l'univers russe et de la langue russe, indépendamment de leur ethnicité. Pour les éleveurs de rennes nénetses de cette région, sont lutsa tous ceux qui n'ont pas de rennes, quelles que soit la langue qu'ils parlent. Ainsi des "Nénetses » peuvent être considérés comme lutsa, même s'ils parlent nénetse, s'ils vivent en ville, n'ont pas de rennes, ou ont adopté des pratiques non nénetses.

51 Historiquement, et cela se produit aujourd'hui encore, les changements de langues ont été fréquents - des Khantys qui passent au nénetse, etc. (Laptander dans ce volume ; Volžanina 2010, p. 59). Mais ceci ne conduit pas forcément à un changement radical de perception de soi : des Nénetses qui sont passés au khanty ou au komi continuent à se dire Nénetses. Nous avons affaire à quelque chose qu'on pourrait appeler nomadisme linguistique, qui se déroule sur plusieurs générations, ou dans la vie d'un seul individu, parfois même avec des mouvements d'aller et retour.

52 Les perceptions peuvent changer ici et là, sous des influences diverses - de l'État ou de l'Église, par exemple - mais tant que les familles migrent avec les rennes dans la toundra, le nénetse demeure en position relativement solide. En même temps, dans les 
villages et dans les villes, il perd rapidement ses locuteurs : c'est une tendance générale que le changement de mode de vie, dans beaucoup d'endroits, ait débouché sur un mouvement à sens unique en direction du monolinguisme russe.

\section{BIBLIOGRAPHIE}

ANDERSON David G., 2000, Identity and Ecology in Arctic Siberia: The Number One Reindeer Brigade, Oxford: Oxford University Press.

ВАВUక̌КIN 1930 = БАБУШКИН А.И., БОЛЬШЕЗЕМЕЛЬСКАЯ ТУНДРА, СЫКТЫВКАР: ИЗДАТЕЛЬСТВО КОМИ ОБСТАТОТДЕЛА.

BALZER Marjorie Mandelstam, 1999, The Tenacity of Ethnicity: A Siberian Saga in Global Perspective, Princeton: Princeton University Press.

BJØRKLUND 2000 = ББЁРКЛУНД ИВАР, «КОЧЕВАЯ ШКОЛА В ТУНДРЕ», in ТОЛКАЧЕВ В.Ф. (РЕД.-СОСТ.), НЕНЕЦКИЙ КРАЙ: СКВОЗЬ ВЬЮГИ ЛЕТ: ОЧЕРКИ. СТАТЬИ. ДОКУМЕНТЫ, АРХАНГЕЛЬСК: ПОМОРСКИЙ ГОСУДАРСТВЕННЫЙ УНИВЕРСИТЕТ ИМЕНИ М. В. ЛОМОНОСОВА, СТР. 485-491.

BLOCH Alexia, 2004, Red Ties and Residential Schools: Indigenous Siberians in a Post-Soviet State. Philadelphia: University of Pennsylvania Press.

BOGOYAVLENSKIY Dmitry, SIGGNER Andy, 2004, “Arctic demography”, in EINARSSON Nils, LARSEN Joan Nymand, NILSSON Annika, and Young Oran R. (eds.), Arctic Human Development Report, Akureyri: Stefansson Arctic Institute, pp. 27-41.

DOLGIH 1970 = ДОЛГИХ БОРИС ОСИПОВИЧ, ОЧЕРКИ ПО ЭТНИЧЕСКОЙ ИСТОРИИ НЕНЦЕВ И ЭНЦЕВ, МОСКВА: НАУКА.

DRAМА 2000 = «ДРАМА ВОРКУТИНСКИХ НЕНЦЕВ», МИР КОРЕННЫХ НАРОДОВ, № 3, СТР. 74-80.

GOLOVNEV Andrei V., OSHERENKO Gail, 1999, Siberian Survival: The Nenets and Their Story, Ithaca: Cornell University Press.

GRÜNTHAL Riho, KOVÁcs Magdolna, 2011, Ethnic and Linguistic Context of Identity: Finno-Ugric Minorities (Uralica Helsingiensia, 5), Helsinki: Tiedekirja.

HABECK Otto, 2005, What it Means to be a Herdsman: The Practice and Image of Reindeer Husbandry among the Komi of Northern Russia, Münster: LIT Verlag.

НОМІС̆ 1976 = ХОМИЧ ЛЮДМИЛА ВАСИЛЬЕВНА, ПРОБЛЕМЫ ЭТНОГЕНЕЗА И ЭТНИЧЕСКОЙ ИСТОРИИ НЕНЦЕВ, ЛЕНИНГРАД: НАУКА.

ISTOMIN Kirill, 1999, "Kolva Volost and Kolva ethnographic group as an example of transition from nomadic to settled way of life. An attempt of analysis", Pro Ethnologia, 8, (Arctic Studies, 3), Tartu: Estonian National Museum, pp. 19-34.

KRIVONOGOV 1997 = КРИВОНОГОВ ВИКТОР ПАВЛОВИЧ, «РУССКО-ЯЗЫЧНЫЕ МЕТИСЫ?», НАРОДЫ СИБИРИ: ПРАВА И ВОЗМОЖНОСТИ, НОВОСИБИРСК: ИЗДАТЕЛЬСТВО ИНСТИТУТА АРХЕОЛОГИИ И ЭТНОГРАФИИ СО РАН, СТР. 148-161. 
LEETE 2013 = ЛЕЭТЕ АРТ, «БОГ ГОВОРИТ НА МОЕМ РОДНОМ ЯЗЫКЕ: ЭТНИЧЕСКАЯ СТРАТЕГИЯ КОМИ ЦЕРКВИ», АНТРОПОЛОГИЧЕСКИЙ ФОРУМ, №18, СТР. 256-275.

LEETE Art, KOOSA Piret, 2012, “The Churches were opened and lots of missionaries arrived: Dialogue between Komi identity and faith", Folklore. Electronic Journal of Folklore, 51, pp. 171-190. LIARSKAYA Elena V., 2009, “Settlement Nenets on the Yamal Peninsula: Who are they?", Folklore. Electronic Journal of Folklore, 41, pp. 33-46.

LIARSKAYA Elena, 2013, "Boarding school on Yamal: History of development and current situation", in KASTEN Erich, DEGRAAF Tjeerd (eds.), Sustaining Indigenous Knowledge: Learning Tools and Community Initiatives for Preserving Endangered Languages and Local Cultural Heritage, Fürstenberg/Havel: Kulturstiftung Sibirien, pp. 159-180.

MAMADYŠSKIJ 1910 = МАМАДЫШСКИЙ Н.Н., УССИНСКИЙ КРАЙ: ПОДВОРНО-ЭКОНОМИЧЕСКОЕ ИССЛЕДОВАНИЕ ПОСЕЛЕНИЙ Р. УССЫ ПЕЧОРСКАГО УЕЗДА В 1909 Г., АРХАНГЕЛЬСК: ГУБЕРНСКАЯ ТИПОГРАФИЯ.

NIGLAS Liivo, 1997, “Reindeer in the Nenets Worldview", Pro Ethnologia, 5, (Arctic Studies, 1), Tartu: Estonian National Museum, pp. 7-33.

PANJUKOV 2012 = ПАНЮКОВ АНАТОЛИЙ, «ИЖМО-КОЛВИНСКАЯ ФОЛЬКЛОРНАЯ ТРАДИЦИЯ: В поискАх истокоВ», АРТ, № 1. Internet : http://www.artlad.ru/magazine/all/598/599/612/625, consulté le $1^{\text {er }}$ novembre 2013.

ŠABAEV Jurij P., 2011, «Les Komis de l'Ižma et les Pomors: deux modèles de transformations culturelles», Études finno-ougriennes, 43, p. 31-72.

STAMMLER Florian, 2005, Reindeer Nomads Meet the Market: Culture, Property and Globalisation at the "End of the Land", Münster: LIT Verlag.

STAMMLER Florian, PESKOV Vladislav, 2008, "Building a 'culture of dialogue' among stakeholders in North-West Russian oil extraction", Europe-Asia Studies, 60(5), pp. 831-849.

TALEEVA L. 2002 = ТАЛЕЕВА ЛЮДМИЛА, МЕТОДИКА ОБУЧЕНИЯ НЕНЕЦКОМУ ЯЗЫКУ КАК ИНОСТРАННОМУ НА НАЧАЛЬНОМ ЭТАПЕ (РАБОТА НА СОИСКАНИЕ СТЕПЕНИ МАГИСТРА, УНИВЕРСИТЕТ ТАРТУ, КАФЕДРА УРАЛЬСКИХ ЯЗЫКОВ). ТАРТУ. Internet: http://www.ut.ee/Ural/ariste/loputood/Talejeva.pdf, consulté le $1^{\text {er }}$ novembre 2013.

TALEEVA M. 2010 = ТАЛЕЕВА МАТРЁНА ИВАНОВНА, ОТЧЁТ О РАБОТЕ ВИЦЕ-ПРЕЗИДЕНТА ПО ВОПРОСАМ ЯЗЫКА, КУЛЬТУРЫ, ОБРАЗОВАНИЯ ТАЛЕЕВОЙ МАТРЁНЫ ИВАНОВНЫ. 2007 - 2010 ГОД. Internet: http:// www.yasavey.org/index.php?

option=com_content\&view=article\&id=181:2012-01-31-07-13-46\&catid=47:ix-\&Itemid=200018, consulté le $1^{\text {er }}$ novembre 2013.

TALEEVA V. 1999 = ТАЛЕЕВА ВАЛЕНТИНА, «ОБРАЗОВАНИЕ В ПОСЕЛКЕ КАРАТАЙКА», БЮЛЛЕТЕНЬ «ЛЬЫОРАВЭТЛЬАН», № 25. Internet: http://www.indigenous.ru/fotki/bull_ru/r_25.htm, consulté le $1^{\text {er }}$ novembre 2013.

TOULOUZE Eva, 1999, "The development of a written culture by the indigenous peoples of Western Siberia", Pro Ethnologia, 7, (Arctic Studies, 2), Tartu: Estonian National Museum, pp. 53-85.

TOULOUZE Eva, 2012, "Forest Nenets folklore and identity", in HASSELBLATT Cornelius, VAN DER HOEVEN Adriaan (eds.), Finno-Ugric Folklore, Myth and Cultural Identity, (Studia Fenno-Ugrica Groningana, 7), Maastricht: Shaker Publishing, pp. 227-240.

TUISKU Tuula, 2001, "The displacement of Nenets women from reindeer herding and the tundra in the Nenets Autonomous Okrug, Northwestern Russia”, Acta Borealia, 18(2), pp. 41-60. 
VALLIKIVI Laur, 2003, « Naître à une vie nouvelle : la conversion au christianisme des éleveurs de rennes nenets ", Études finno-ougriennes, 35, p. 7-42.

VALLIKIVI Laur, 2005, “Two wars in conflict: Resistance among Nenets reindeer herders in the 1940s", Studies in Folk Culture, 5, Tartu: Tartu University Press, pp. 14-54.

VALLIKIVI Laur, 2009, “Christianisation of words and selves: Nenets reindeer herders joining the state through conversion", in PELKMANS Matthijs (ed.), Conversion After Socialism: Disruptions, Modernities and the Technologies of Faith, Oxford, New York: Berghahn, pp. 59-83.

VALLIKIVI Laur, à paraître, "Becoming Christian and Russian: Mission encounters and identity practices among the Nenets reindeer herders", in GRAY Patty, PLATTET Patrick, VATÉ Virginie (eds.), Doing Religion in the Russian North: Uses of Religiosity after Socialism, New York, Oxford: Berghahn. VOLŽANINA $2010=$ ВОЛЖАНИНА ЕЛЕНА АЛЕКСАНДРОВНА, ЭТНОДЕМОГРАФИЧЕСКИЕ ПРОЦЕССЫ В СРЕДЕ НЕНЦЕВ ЯМАЛА В ХХ - НАЧАЛЕ ХХІ ВЕКА, НОВОСИБИРСК: НАУКА.

WIGET Andrew, BALALAEVA Olga, 2007, “Crisis, conversion, and conflict: Evangelical Christianity, rapid change, and the Eastern Khanty”, Sibirica 6(1), pp. 1-29.

ŽURAVLEVA 2000 = ЖУРАВЛЕВА Т.Ю., «К ВОПРОСУ ОБ ОРГАНИЗАЦИИ И ФУНКЦИОНИРОВАНИИ КОЧЕВОЙ ШКОЛЫ В ОБЩИНЕ НЕНЦЕВ-ЕДИНОЛИЧНИКОВ ЯМБТО», in САВЕЛЬЕВА Э.А. (РЕД.), КОРЕННЫЕ ЭТНОСЫ СЕВЕРА ЕВРОПЕЙСКОЙ ЧАСТИ РОССИИ НА ПОРОГЕ НОВОГО ТЫСЯЧЕЛЕТИЯ: ИСТОРИЯ, СОВРЕМЕННОСТЬ, ПЕРСПЕКТИВЫ, СЫКТЫВКАР: КОМИ НАУЧНЫЙ ЦЕНТР УРО РАН, СТР. 524-527.

\section{NOTES}

1. Il est toujours difficile de rendre l'adjectif мАлочисленный, qui veut dire peu nombreux. Cette expression ne convient pas pour un singulier collectif tel que «peuple ». L'adjectif « petit ", dans ce contexte, ne reflète aucune nuance péjorative. Dans ce contexte, il renvoie à un statut défini par la loi, dont l'un des critères est un nombre de ressortissants inférieur à 50000 . (NdT)

2. Le nombre des Nénetses, d'après les derniers recensements en Russie, est le suivant : 1979 27294,1989 - 34 190, 2002 - 41 302, 2010 - 44640.

3. Si au recensement de 2002 au moins 28128 Nénetses ont marqué qu'ils connaissaient leur langue (dans les régions des « petits » peuples du Nord - les districts autonomes nénetse, iamalonénetse, khanty-mansi, du Tajmyr, et la République komie), en 2010 ils n'étaient plus que 19567 (dans toute la Russie). Ainsi, aujourd'hui, moins de la moitié des Nénetses (43,8 \%) maîtrisent leur langue traditionnelle. Les variations régionales peuvent être considérables. Par exemple en 2010, dans le district autonome nénetse, sur 7504 Nénetses, seuls 750 ont marqué qu'ils connaissaient la langue, soit $10 \%$. La majorité de ceux qui maîtrisent la langue vivent dans le district autonome iamalo-nénetse ( 16390 , soit $55,1 \%$ des Nénetses de la région) et dans le kraï de Krasnojarsk (1650, soit 45,4\%), où se trouvent les communautés les plus vivaces d'éleveurs de rennes nomades. (http://www.perepis2002.ru/; http://www.perepis-2010.ru/, consulté le $1^{\mathrm{er}}$ novembre 2013).

4. Nous ne disposons d'aucun chiffre exact sur le nombre de Nénetses nomades. On dispose d'évaluations par région. Ainsi, dans le district autonome iamalo-nénetse, environ 14500 sur toute la population autochtone (y compris les Khantys éleveurs de renne) sont engagés dans un mode de vie nomade (Stammler 2005, p.5; cf. aussi Bogoyavlenskiy, Siggner 2004, p. 31 ; Volžanina 2010). L'un des noyaux de la vie nomade est la péninsule de Jamal, où la société nomade s'est avérée la plus vitale : le nombre total des nomades (environ 5 500) est resté plus ou moins au niveau de la période précédant la collectivisation (Liarskaya 2009, p. 34, 44 note 2). 
Toutefois dans les toundras européennes, le nombre des familles nomades a régressé considérablement depuis le milieu du $\mathrm{xx}^{\mathrm{e}}$ siècle. On estime à 1500 le nombre d'éleveurs nomades dans le district autonome nénetse, sans compter ceux qui sont enregistrés dans la République komie (Stammler, Peskov 2008, p. 836). Ce dernier chiffre semble néanmoins gonflé. En résumé, on peut suggérer que dans l'ensemble, le tiers des Nénetses vit en permanence dans la toundra.

5. Aujourd'hui, il n'y a plus de kolkhozes proprement dits, mais des structures coopératives, des structures d'État ou des structures privées qui en découlent ; les deux premiers types conservent dans la langue parlée les mêmes noms qu'à l'époque soviétique.

6. En russe : ЧУМРАБОТНИЦА.

7. Les Nénetses essayèrent autant que possible d'échapper à ce nouveau système. Par exemple dans le kolkhoze appelé Harp (en nénetse : Aurore boréale) à l'ouest de la toundra de la Grande Terre, le nouveau système de roulement était en place sur le papier, mais, surtout au début de l'opération, pendant les années 1960 et 1970, une partie des familles continuaient à vivre ensemble dans la toundra (Tuisku 2001, p. 46-47).

8. D'après K. P. Taleeva, institutrice à Nel'min-Nos, sur les dix enfants commençant l'école en 2000, seuls deux étaient capables de parler en nénetse de la vie quotidienne (L. Taleeva 2002, p. 6-7).

9. Lors du dernier recensement (celui de 2010), il y avait déjà $24,1 \%$ des Nénetses (10 774 personnes) qui considéraient le russe comme leur langue maternelle (Родной язык). Presque tous les autres considéraient comme langue maternelle le nenetse (32 640 Nénetses, soit 73,1\%), même si le pourcentage de ceux qui maîtrisent réellement la langue est sensiblement plus faible (http://www.perepis-2010.ru/, consulté le $1^{\mathrm{er}}$ novembre 2013).

10. En russe : НАЦИОНАЛЬНЫЙ ПОСЕЛОК.

11. En 2002, sur les 237 enfants nénetses qui vont à l'école de Nel'min-Nos, 52 ont indiqué qu'ils connaissaient le nénetse. Mais ce chiffre ne reflète vraisemblablement pas la réalité, il est exagéré. (Ces données reposent sur la "micro base de données" du recensement de 2002 http://std.gmcrosstata.ru/webapi/jsf/tableView/customiseTable.xhtml, consulté le $1^{\mathrm{er}}$ novembre 2013. Les informations peuvent ne pas être exhaustives, dans la mesure où les catégories ayant été choisies par un tout petit nombre de personnes n'y figurent pas. En 2002, les questions posées à propos de la langue étaient les suivantes : Est-ce que vous maîtrisez le russe? Quelles autres langues connaissez-vous ? À cette dernière question, il était possible de répondre en indiquant trois langues.)

12. La non-maîtrise de la langue dominante est un paramètre éloquent. Si nous comparons les données des derniers recensements sur la non-maîtrise du russe parmi les Nénetses, nous voyons que le nombre des Nénetses ne maîtrisant pas le russe est en diminution, lente mais certaine : en 2002, 4607 personnes n'ont pas coché la case montrant qu'ils savaient le russe $(11,1 \%)$, alors qu'en 2010 ils ne sont plus que 3827 (8,6\%). Parmi les peuples du nord de la Russie, c'est chez les Nénetses que l'on trouve la proportion la plus élevée de personnes non russophones, ce qui renvoie à l'existence de communautés nénetsophones fort vivaces (http://www.perepis2002.ru/ ; http://www.perepis-2010.ru/, consulté le $1^{\mathrm{er}}$ novembre 2013).

13. Ici, « russe » ne renvoie pas forcément à une ethnicité, mais à un mode de vie et de culture caractérisé par l'usage de la langue russe, ce qui peut inclure des Ukrainiens, des Tatars, etc.

14. Il s'agit de baptistes conservateurs et fondamentalistes, qui passèrent en 1961 dans la clandestinité, car à la différence d'autres baptistes contrôlés par l'État, ils refusaient les ingérences du pouvoir et notamment l'obligation de s'enregistrer. Ils font partie aujourd'hui de l'Union internationale des Églises des chrétiens baptistes évangéliques (МЕЖДУНАРОДНЫЙ СОЮЗ ЦЕРКВЕЙ ЕВАНГЕЛЬСКИХ ХРИСТИАН-БАПТИСТОВ), qui rassemble les croyants russophones. Avec la chute du pouvoir soviétique ils sortent de la clandestinité et font ouvertement œuvre de prosélytisme. Ils sont intégrés dans le monde russe et sont russophones : pour les Nénetses, malgré leur propre marginalité, ils ouvrent la voie à l'univers russe environnant. 
15. Même dans les générations plus âgées, on trouve quelques individus, pour la plupart des femmes arrivées dans le groupe par mariage, qui sont allés quelques temps à l'école et qui connaissent le russe. De même, en 1983, deux enfants de Jamb-to ont été pris à l'insu de leurs parents par un hélicoptère et ils ne sont rentrés que huit ans plus tard. Mais ce sont là des exceptions.

16. En fait, dans le village de Karatajka, bien que la plupart des habitants soient d'origine nénetse, la principale langue de communication est le komi. Cet enseignant maîtrise le nénetse, le russe et le komi. Le nénetse a peu de prestige et il semble que les enfants nenetses aient honte de l'utiliser (cf. V. Taleeva 1999). Un Nénetse de Jamb-to m'a avoué avoir appris, à l'école de Karatajka, le komi avant le russe.

17. Il avait été le premier étranger à rendre de courtes visites aux Nénetses de Jamb-to.

18. En russe : ЛЕТНЯЯ КОчЕВАЯ ШКОЛА.

19. Vorkuta, la ville la plus septentrionale de la République komie, est presque intégralement russophone. En même temps, on trouve également dans la République komie des protestants qui valorisent l'usage des langues locales (pour plus de détails, cf. Leete 2013, Leete, Koosa 2012, sur les églises dans le sud de la République komie, qui fonctionnent en komi).

20. Andrew Wiget et Olga Balalaeva soulignent l'influence des missionnaires baptistes russes sur l'idéologie linguistique des Khantys : "When they sing and pray, they do so in Russian, because as the [Khanty] elder, V.G., told us, “Khanty language doesn't have words to talk about these things." » $(2007$, p. 8$)$.

21. Naturellement, les jeunes Nénetses qui étaient allés à l'école et dont le russe était meilleur que mon nénetse, préféraient parler russe avec moi. Il était aussi parfois difficile d'avoir de l'aide de la part des jeunes dans mon apprentissage du nénetse. L'un d'entre eux m'a dit que cela n'avait aucun sens d'apprendre le nénetse, de toute manière cela ne servirait à rien.

22. Ailleurs dans l'Arctique russe, chez les éleveurs de rennes kolkhoziens, le russe a pénétré bien plus en profondeur. Par exemple Anderson (2000, p. 30-31) donne l'exemple de la brigade des Evenks du Tajmyr où l'on parle evenk. "To distinguish the various tasks of domestication, it is significant that the herders of the Number One Brigade speak of reindeer in a variety of languages. When comparing the attitude, markings, or genealogy of a particular reindeer, they prefer to use the Evenki language, which is rich in such distinctions [...]. When speaking of how a reindeer is best used, how it is best classified as taught or untaught, harnessable or wild, or as the charge of a particular person (or just a 'farm' reindeer) the herders tend to use the language of Soviet state pastoralism - Russian. When using Evenki, the herdsmen rarely use a word which identifies a single population of reindeer as having a fixed and set identity (but may use the Russian word stado [herd] for this idea). They instead speak actively of 'bringing reindeer together' [takkakhin-mi] ». Pour les éleveurs nénetses privés, tout ce qui est en rapport avec l'élevage du renne est avant tout pensé et parlé en nénetse.

23. D'après le recensement de 2010, 828 (2\%) Nénetses considèrent le komi comme langue maternelle (la plupart d'entre eux, 508, résident dans le district autonome nenets). Environ autant de Nénetses (803) affirment savoir le komi.

24. "Nénetse » en komi. Les Nénetses nénetsophones ont repris cette appellation aux Komis de l'Ižma, qui, eux, utilisent le terme « Jaran » pour désigner tous les Nénetses.

25. Certains Nénetses zyriénisés ne sont jamais passés par Kolva, même s'ils sont appelés, par extensions, « Jaran de Kolva » (cf. Panjukov 2012).

26. Une partie des Nénetses s'est fondue parmi les Iz'vatas depuis très longtemps. Toutefois, de nos jours, il se trouve des individus conscients de leur origine nénetse (par l'intermédiaire de la population de Kolva), ne serait-ce que par le nom, et qui se sentent «intermédiaires » entre les Komis et les Nénetses (Habeck 2005, p. 68).

27. Avant d'être officialisés, les Nénetses de Jamb-to utilisaient des pâturages attribués à des kolkhozes ou des sovkhozes dont la majorité était komiphone. 
28. Habeck, qui a étudié les Iz'vatas, a noté que « ... for the Izhma Komi, Nenets identity relates to concepts of experience, knowledge and the quality of being a good reindeer herder» (2005, p. 68).

\section{RÉSUMÉS}

Cet article analyse le lien particulier entre le mode de vie et la langue dans une période de mutations culturelles rapides. Les Nénetses sont la communauté nomade la plus résistante dans l'Arctique russe, ce qui se reflète dans son usage plus large qu'ailleurs de la langue nénetse. Pourtant, ces derniers temps, la langue se perd à un rythme accéléré - différent suivant les communautés. La thèse centrale est que la vie avec les rennes permet aux Nénetses de garder leur langue. L'idéologie linguistique des Nénetses est fondée sur le pragmatisme et la situativité, comme le montrent les changements de langue consécutifs au changement d'environnement physique et social. Je me concentrerai sur deux communautés non soviétisées et partiellement christianisées, qui permettront d'illustrer différentes dimensions de l'usage linguistique et plus largement des évolutions culturelles dans la région.

Artikkel analüüsib spetsiifilist seost eluviisi ja keele vahel kiirete kultuuriliste muutuste ajal. Neenetsite näol on tegemist elujõulisima nomaadikogukonnaga Vene Põhja-aladel ja sellest tulenevalt on ka oma keele kasutamine laialdasem kui mujal. Siiski on 21. sajandi algul keele kadu osutunud kiirenevaks - erinevates piirkondades küll erinevalt. Artikli keskne tees on, et põhjapõtradega elamine võimaldab neenetsitel säilitada oma keelt ja et neenetsite keeleideoloogia soosib pragmaatilisust ja situatiivsust, mida tõendavad sagedased keelevahetused füüsilise ja sotsiaalse elukeskkonna muutudes. Vaatluse all on kaks sovietiseerimata ja hiljuti osaliselt ristiusustatud rändkogukonda, mis näitlikustavad neenetsite keelekasutuse suurt paindlikkust ja seonduvaid kultuurilisi protsesse regioonis laiemalt.

This paper analyses a specific link between the way of life and the use of language during rapid cultural transformations. The Nenets have the largest nomadic community in Arctic Russia. As a result, they have retained their language better than most other indigenous peoples in the region. Nevertheless, at the early 21st century, there has been a quick language loss, although at a different pace in different areas. The main thesis here is that those Nenets who live with reindeer are motivated to use Nenets. Their language ideology favours pragmatic and situative approach to language, as it is proved by language shifts when the physical and social environment alters. The ethnographic focus is on two non-Sovietised and partly Christianised Nenets communities that show a significant flexibility of language use. 
INDEX

Mots-clés : élevage du renne, Christianisme, nomadisme, Baptistes, bilinguisme

motscleset põhjapõdrakasvatus, Ristiusk, nomadism, Baptistid, kakskeelsus

nomsmotscles Evenks, Khantys, Komis de l'Ižma, Nénetses de la toundra, Nénetses de Jamb-to, Nénetses de l'Oural, Russes

Index géographique : Krasnoïarsk (kraï), Komi (République), Sovetskij, Vorkuta, Kolva, Kanin, Karatajka, Nar'jan-Mar, Nelmin-Nos, Toundra de la Petite Terre, Toundra de la Grande Terre, Ust'-Kara, Sibérie, Sibérie occidentale, Iamalo-Nénétsie (Yamalie) (district autonome), Taïmyr (presqu'île de), Nénetses (district autonome), Khanty-Mansiïsk (Ougrie) (district autonome) disciplines nénetse

Keywords : Bilingualism, Christianity, Baptists, Nomadism, Reindeer-herding

Thèmes : sociolinguistique 